\title{
Using a Design Workshop to Explore Accessible Ideation
}

\author{
Cynthia L. Bennett ${ }^{1}$, Kristen Shinohara ${ }^{2}$, Brianna Blaser ${ }^{3}$, Andrew Davidson ${ }^{1}$, Kat M. Steele ${ }^{4}$ \\ ${ }^{1}$ Human Centered Design and Engineering, ${ }^{2} \mathrm{iSchool},{ }^{3} \mathrm{DO}-\mathrm{IT}$, ${ }^{4}$ Mechanical Engineering \\ University of Washington, Box 352315 \\ Seattle, WA 98195 \\ \{bennec3, kshino, adavid7, blaser, kmsteele\}@uw.edu
}

\begin{abstract}
Although a critical step in the technology design process, ideation is often not accessible for people with disabilities. We present findings from a design workshop facilitated to brainstorm accessible ideation methods. Groups, mostly engineers, ideated on a design challenge and documented access barriers encountered by participants with disabilities. They then ideated and prototyped potential solutions for decreasing access barriers. We offer suggestions for more accessible communication and ideation on a design team and insights from using a workshop as a site for rethinking ideation.
\end{abstract}

\section{Keywords}

Accessibility; Brainstorming; Design; Ideation; Disability

\section{INTRODUCTION}

Ideation, also known as brainstorming, is a crucial phase of the technology design process used to inspire and to refine designs. However, few projects have explored how to make ideation itself more accessible for people with disabilities. For example, many students are taught to ideate by sketching, but this method may be difficult for people with vision or mobility impairments. As efforts to increase the number of people with disabilities in computing and accessible products continue, it is imperative to explore how ideation can both be made more accessible for all designers and co-design participants.

We explored this question during a short design workshop, by prompting engineers, and participants with disabilities, some being engineers, to identify access barriers during an initial design challenge and to then prototype potential methods for making ideation more accessible. We offer two contributions: (1) We present accessible communication and ideation considerations prototyped during the design activity, and (2) We offer a design activity as a generative site for documenting and improving upon design-related access barriers. We hope designers can use these preliminary findings to ideate more accessibly and to motivate research on increasing the accessibility of the design process.

\section{RELATED WORK}

Researchers have introduced strategies for designing for people with disabilities such as Ability-Based Design [10], Design for Social Accessibility [6], and User Sensitive Inclusive Design [5]. One popular framework, Universal Design [8], advocates designing for the most people to have access, including those with disabilities. However, these strategies lack specific methods for accessibly navigating various phases of the design process.

Permission to make digital or hard copies of part or all of this work for personal or classroom use is granted without fee provided that copies are not made or distributed for profit or commercial advantage and that copies bear this notice and the full citation on the first page. Copyrights for third-party components of this work must be honored. For all other uses, contact the Owner/Author.

Copyright is held by the owner/author(s).

ASSETS '16, October 23-26, 2016, Reno, NV, USA

ACM 978-1-4503-4124-0/16/10.

http://dx.doi.org/10.1145/2982142.2982209
Notably, Larsen et al [4] recommend ideation strategies for codesigning with children who do not speak, and Bueler et al [2] offer recommendations for teaching a 3D-printing course with students with and without intellectual disabilities. Design workshops are also a popular tool for introducing people to the design process. For example, IDEO offers a free toolkit [3] to K12 educators on incorporating design thinking into curricula. The Hasso Plattner Institute of Design at Stanford introduces students to each phase of the design process [7] in a short workshop. Our approach was similar, as groups briefly ideated on a design challenge. However, we narrowed the challenge to focus on ideation to gather more targeted accessibility suggestions.

\section{METHODS}

Procedure. The 90-minute design workshop was conducted during a Capacity Building Institute sponsored by AccessEngineering [1] that gathered engineering educators, engineers, and disability service professionals, some of whom had disabilities. Everyone was introduced to the phases of the design process, with extra background on ideation methods. Each group was given a bag of supplies including sticky notes, pens, felt, pipe cleaners, popsicle sticks, Play-Doh, and other craft supplies. Groups of participants were asked to ideate solutions for making smart classrooms more accessible by first brainstorming several solutions and then choosing one for ideating refinements. Participants then reflected by identifying access barriers they encountered in this process, what went well, and what was challenging. Each group then chose one access barrier they encountered and ideated and prototyped potential solutions. Participants engaged in another reflection and presented their findings to the group. Data include a CART (communication access real-time translation) transcript of the activity, reflection sheets from each group, and field notes from two researchers. All data were thematically analyzed, and we present these findings along with solutions prototyped during the workshop.

Participants. We had about 40 participants divided into seven groups, with at least one member with a disability in each. Four workshop attendees offered to serve as the person with a disability for their group, and the remaining three were volunteers from the community. Three participants had vision impairments, one had a hearing impairment, one identified as neurodiverse, one had a learning disability, and one had physical disabilities and attended via a Beam remote presence robot [9].

\section{FINDINGS}

Communication. An emergent theme across groups was access barriers impacting communication during the workshop. Ideation is rooted in individuals sharing and building upon others' ideas; however, traditional ideation methods created persistent challenges. For example, conversation was difficult for deaf or hard of hearing participants to follow and contribute to the group. These participants expressed frustration lip reading or watching an interpreter during a fast paced conversation while also examining sketches or other artifacts. One group passed around a talking stick to discourage interrupting and to offer each member 
a chance to share. A participant from a different group realized that "each individual member of the group has a particular strength or weakness." They categorized preferences such as writer, speaker, and tactile in a matrix and asked each member to fill out their strengths, which were then used to allocate roles during the workshop. Groups realized that different communication styles and access needs prevented everyone from maintaining awareness and contributing. As demonstrated in the above examples, they found systematic and multimodal methods of communication helpful to ensuring that everyone could share their ideas.

Ideation. Beyond communication, the groups identified additional solutions for more accessible ideation. One group with a neurodiverse participant noted difficulty following the progression of ideas as they were tossed into a pile in the middle of the table. They designed a pattern and participants took turns placing ideas so it was easier to track the trajectory of the ideation session. Vision-impaired participants were unaware of sketched ideas and handwritten sticky notes as they piled on tables. In response, one group mocked up a Play-Doh design for a system to synchronize handwritten and typed ideas for participants to read visually or with audio. The group also noted the potential for such systems to better include remote collaborators such as people attending via a Beam. One group tried to make 2D ideas more accessible non-visually by adding textures to sketches, and cutting sticky notes into different shapes to categorize ideas tactually.

Workshop. To gather formative insights, we positioned a design workshop as a site for documenting access barriers and thinking of design solutions. Not only did groups think of creative ways to be more inclusive while ideating, the workshop itself prompted participants to rethink design. One participant concluded his group's presentation with, "Even in the [design] process, you have to consider accessibility. That was my aha moment." Positioning participants as co-designers in a workshop setting raised awareness about access barriers for some and helped them to think of quick solutions to try in their own design environments. However, the workshop itself contained access barriers. For example, hearing-impaired participants were unable to focus on interruptions by the facilitator and their groupmates at the same time. These access barriers raised an interesting tension around purposefully not making the workshop as accessible as it could have been to inspire ideas, though we did not do anything explicitly to make the workshop less accessible. While experiencing access barriers allowed them to be identified and used for ideating more accessible methods, we should consider how to most thoughtfully do this so not to position participants to have a negative experience. A potential solution is to incorporate accessibility considerations often not implemented during similar design workshops, such as visual and audio cues to, allow people to finish their thoughts before a facilitator speaks. Participants could reflect on how accessibility considerations impacted collaboration, which could then be used to begin refining existing solutions or generating new ones.

\section{CONCLUSION}

We presented findings from a workshop aimed at designing ways to ideate more accessibly. During the activity, participants found several access barriers to communication and ideation which prevented participants with disabilities from contributing equally. In response, they used common craft supplies, often employed during prototyping phases of the design process, to ideate more accessibly. The variety of supplies and multimodal communication methods seemed to provide more opportunities for greater participation by everyone. Though more work should be done to insure the workshops themselves are accessible, the setting built on [2] and [4] and allowed participants to immediately try solutions positioning it as a promising method for further improving design process accessibility.

\section{ACKNOWLEDGMENTS}

Thanks to our participants and AccessEngineering (NSF grant number EEC-1444961).

\section{REFERENCES}

[1] AccessEngineering. Overview. http://uw.edu/doit/programs/accessengineering/overview Retrieved 6/23/2016.

[2] E. Buehler, W. Easley, S. McDonald, N. Comrie, and A. Hurst. 2015. Inclusion and Education: 3D Printing for Integrated Classrooms. In Proceedings of the 17th International ACM SIGACCESS Conference on Computers \& Accessibility (ASSETS '15). ACM, New York, NY, USA, 281-290. DOI=http://dx.doi.org/10.1145/2700648.2809844

[3] IDEO. Design Thinking Toolkit for Educators. https://www.ideo.com/work/toolkit-for-educators Retrieved 6/22/2016.

[4] H. S. Larsen and P. L. Hedval. 2012. Ideation and ability: when actions speak louder than words. In Proc. PDC '12, Vol. 2. ACM, New York, NY, USA, 37-40. DOI=http://dx.doi.org/10.1145/2348144.2348157

[5] A. Newell, P. Gregor, M. Morgan, G. Pullin, and C. Macaulay. 2011. User-Sensitive Inclusive Design. Universal Access in the Information Society. 10, 3, 235243.

[6] K. Shinohara and J. O. Wobbrock. 2011. In the shadow of misperception: Assistive technology use and social interactions. In Proc. CHI '11 (Vancouver, BC), 705-714. http://doi.acm.org/10.1145/1978942.1979044

[7] Stanford University Institute of Design. Design Project Zero: A 90 Minute Activity. http://dschool.stanford.edu/use-our-methods/designproject-zero-a-90-minute-experience/ Retrieved 6/22/2016

[8] C. Stephanidis, D. Akoumianakis, M. Sfyrakis, and A. Paramythis. 1998. Universal accessibility in HCI: Processoriented guidelines and tool requirements. In Proc. User Interfaces for All' '98.

[9] Suitable Technologies Beam Presence Smart System. https://suitabletech.com/beampro/ Retrieved 6/22/2016.

[10] J. O. Wobbrock, S. K. Kane, K. Z. Gajos, S. Harada, and J. Froehlich. 2011. Ability-based design: Concept, principles, and examples. ACM TACCESS. 3, 3, 1-27. http://doi.acm.org/10.1145/1952383.195238 